\title{
Médiévales
}

Langues, Textes, Histoire

74 | printemps 2018

Chanter la Croisade albigeoise

\section{Quand la chanson de croisade se compose en cycle. Expériences d'écritures françaises et occitanes}

When the Crusade Song is Composed in Cyclic Form. French and Occitan

Experiences of Writing

\section{Marjolaine Raguin-Barthelmebs}

\section{OpenEdition}

\section{Journals}

Édition électronique

URL : https://journals.openedition.org/medievales/8412

DOI : $10.4000 /$ medievales. 8412

ISSN : $1777-5892$

Éditeur

Presses universitaires de Vincennes

Édition imprimée

Date de publication : 15 juillet 2018

Pagination : 45-60

ISBN : 978-2-84292-837-7

ISSN : 0751-2708

\section{Référence électronique}

Marjolaine Raguin-Barthelmebs, «Quand la chanson de croisade se compose en cycle. Expériences

d'écritures françaises et occitanes », Médiévales [En ligne], 74 I printemps 2018, mis en ligne le 15 juillet 2019, consulté le 24 avril 2022. URL : http://journals.openedition.org/medievales/8412 ; DOI : https://doi.org/10.4000/medievales.8412 
Marjolaine Raguin-Barthelmebs

\section{Quand la chanson de croisade se compose en cycle Expériences d'écritures françaises et occitanes}

Dans le cadre d'une réflexion d'ensemble sur la Chanson de la Croisade albigeoise, il semble intéressant, pour ne pas dire nécessaire, de porter attention, au-delà de ce seul texte, à un élément majeur à nos yeux, et qui l'inscrit dans une généalogie et une tradition générique et textuelle des chansons de croisade en français et en occitan : leur caractère cyclique ${ }^{1}$.

Rappelons d'abord que l'on compose, dans ces deux langues, des chansons de croisade lyriques et des chansons de croisade épiques. C'est à ces dernières que nous nous intéresserons ici. Notre réflexion s'attache à la poétique de la chanson de croisade épique et à son articulation cyclique, et non au détail de chaque texte. Nous proposons donc de réfléchir à l'acte qui consiste à écrire la chanson de croisade épique ${ }^{2}$, en français et en occitan, dans le cadre d'un cycle. Elle est la geste qui chante des événements contemporains.

Il est en effet intéressant de noter que ce genre littéraire, entre geste et chronique, s'inscrit dans l'une et l'autre de ces catégories narratives par une série d'éléments. À la narrativité de la chronique s'attachent l'emploi d'une temporalité précise, le récit d'une histoire relativement contemporaine, et un souci d'exactitude fondée sur un témoignage. À la chanson de geste, récit de l'héroïsme ${ }^{3}$ d'abord historique et politique, s'attachent la mise en scène glorieuse des générations de pères/pairs, l'exaltation chevaleresque et nobiliaire, l'insistance sur les valeurs seigneuriales, la grandiloquence des batailles, la démesure, le souffle épique et ce que sont, à mes yeux en

1. Une première version de cette réflexion a été exposée lors de la journée d'étude « The Crusades in France et Occitania. Roots Impact, and Cultural Signification of the Crusading Movement », organisée au Royal Holloway, University of London, le 10 septembre 2016.

2. Sur le triptyque lyrique/épique/dramatique et l'erreur dont il procède, ou sur les genres et les types littéraires, voir G. GENETTE, Introduction à l'architexte, Paris, 1979.

3. P. ZuMTHOR, Essai de poétique médiévale, Paris, 1972, p. 324. 
tout cas, les deux grandes caractéristiques de l'épique et qui marquent pour l'auteur la volonté d'y inscrire son récit : l'emploi d'un certain vocabulaire corrélé à des motifs et des formules dites épiques ${ }^{4}$, et le rattachement à une tradition de récits épiques qui a pour conséquence la création de cycles ces cycles épiques qui nous intéressent ici. Nous retenons que les chansons de croisade épiques, textes de poésie narrative, s'attachent pleinement à la chanson de geste et rappelons la critique de Robert Francis Cook, s'interrogeant sur le caractère épique du cycle de la croisade ${ }^{5}$. Elles en ont l'expression formulaire et la tendance à la parataxe, le vers à coupe centrale souvent alexandrin, les laisses monorimes, les épithètes, l'onomastique, les interventions régulières du jongleur, les annonces et les rappels ${ }^{6}$.

La chanson de croisade épique a pourtant une dimension historique certaine, et est reçue par les historiens, avec quelque précaution, comme document d'histoire ; certains ayant parfois du mal à comprendre que, s'agissant avant tout d'un texte littéraire, l'exagération épique et les nécessités de soudure des événements ou des mains entre eux sont signes de la littérarité du texte et des procédés de l'auteur, n'invalidant pas son témoignage en tant que tel et étant caractéristique en définitive de la nature de la source. La chanson de croisade épique, moins encore que la lyrique, n'est pas l'histoire mais l'écriture de celle-ci. Avec ses codes, ses procédés... et les attentes de son public.

Elle renseigne à coup sûr sur les événements, les tendances idéologiques des partis en présence, le nom des personnages impliqués, et cela avec une bonne fiabilité, pourtant le gros de ses estimations chiffrées par exemple ou certains de ses faits doivent être considérés avec prudence.

De ces cycles épiques, nous avons retenu les cycles de croisade. Ceux dont on signale pour le français, depuis Bertrand de Bar-sur-Aube au début du XIII ${ }^{\mathrm{e}}$ siècle, un triptyque composé du cycle du roi - en particulier la Chanson de Roland, le Pèlerinage de Charlemagne, et la Chanson d'Aspremont -, du cycle de Garin de Monglane plus connu comme celui de Guillaume d'Orange - en particulier le Charroi de Nîmes, le Couronnement de Louis, la Chanson de Guillaume -, et du cycle de Doon de Mayence ou des vassaux rebelles - Raoul de Cambrai, Renaut de Montauban, la

4. Cf. J.-P. MARTIN, Les Motifs dans la Chanson de geste : définition et utilisation (discours de l'épopée médiévale, I), Paris, 2017 ; édition reprise et complétée du volume du même auteur en 1992.

5. R. F. COOK, «Chanson d'Antioche », chanson de geste : le cycle de la croisade est-il épique?,Amsterdam, 1980, p. 5-8.

6. Ibid., p. 12. Leur degré d'exactitude historique est variable, certainement plus haut pour les textes occitans peu ou pas remaniés, ou pour le noyau dur du premier cycle français de la croisade, plus diffus voire inexistant pour les enfances et autres... Néanmoins, c'est là somme toute le cas, exactement comme pour le reste du corpus des chansons de gestes : on peut, à certains égards, estimer que la Chanson de Roland conserve plus du souvenir historique que Mainet. 
Chevalerie Ogier. On y adjoint souvent un cycle des Lorrains fait de violence et d'anarchie avec notamment Garin le Loherain.

Pour la chanson de geste en général, l'écriture cyclique n'est pas celle - schématisons - d'un roman fleuve en plusieurs tomes, elle s'y oppose même ${ }^{7}$. Pensée au fur et à mesure par des auteurs successifs, voire étant un effet de la réception et du regroupement d'œuvres aux thématiques semblables, la cohérence et la concordance de la matière et des événements des chansons d'un même cycle n'est pas totale. Ainsi, dans le cycle de Guillaume, Vivien meurt plusieurs fois et les versions de sa mort ne concordent pas : $c f$. la Chanson de Guillaume, la Chevalerie Vivien ou Aliscans.

Paul Zumthor, dans son Essai de poétique médiévale ${ }^{8}$, s'appuyant sur les travaux de Jean Frappier ${ }^{9}$, souligne que 1'un des facteurs qui contribua certainement à la formation de cycles épiques est la création d'agents épiques par les chansons. Lesquels agents possèdent leurs caractéristiques propres, sortes de types, élaborés et définis plus étroitement au fil de la tradition littéraire afférente, et fonctionnent comme autant de «types-cadres» dépendant de propositions narratives peu variées. Ceux-là deviennent des « personnages » autour desquels se constituent des cycles et dont ils sont le noyau et l'élément structurant. Zumthor, parlant dans le même ouvrage des cycles épiques français, ne mentionnera jamais celui de la croisade ${ }^{10}$.

Dans leur Que sais-je? sur La Littérature française du Moyen Âge, Armand Strubel et Dominique Boutet écrivaient, à la fin du point consacré à l'écriture cyclique dans la chanson de geste, qu'il s'agit « d'un phénomène purement littéraire, aux antipodes des préoccupations des jongleurs ${ }^{11} \gg$. Il ne peut s'agir de cela. Il s'agit certes d'un phénomène littéraire - mais qu'entend-on par-là exactement ? La perception par l'auditoire ou le public de l'appartenance cyclique d'un texte est certainement une préoccupation de tous les auteurs et de tous les interprètes ou «performeurs » du texte. En effet, ils ont un intérêt évident à ce que celui-ci soit reconnu comme s'inscrivant dans une grande histoire, une tradition qui augmentera l'attention du public et favorisera un accueil attentif sinon bienveillant.

L'écriture cyclique des chansons de croisade épiques n'est pas qu'un effet de la réception : le poète lui-même, ou le remanieur, inscrit le

7. La réflexion semble être la même pour la littérature de genre contemporaine: on verra A. BESSON, D'Asimov à Tolkien : cycles et séries dans la littérature de genre, Paris, 2004.

8. P. ZuMTHOR, Essai de poétique médiévale..., p. 325.

9. J. FRAPPIER, Les Chansons de geste du cycle de Guillaume d'Orange, Paris, t. II, 1965 (le tome I ayant été publié en 1955).

10. P. ZuMTHOR, Essai de poétique médiévale..., p. 463-465.

11. D. Boutet et A. Strubel, La Littérature française du Moyen Âge, Paris, 1997, p. 21. 
plus souvent son texte dans une généalogie littéraire, que nous désignons aujourd'hui par cycle $e^{12}$, et parfois le revendique explicitement. Voilà déjà une première considération essentielle. C'est la continuité qui s'établit d'une chanson à l'autre, qui fait le cycle. Celle-ci peut être formelle et toucher aux procédés stylistiques de composition et au vocabulaire, ou liée au fond du texte et en reprendre le sujet et l'argument, que la chanson déclinera et recomposera. Il ne s'agit pas seulement de faire le récit des mêmes événements autrement, ou même de les «continuer » sous forme de réécriture ou de suite, mais de lier le récit à celui d'un prédécesseur. L'établissement de ce lien prendra la forme, par exemple, d'un récit de croisade articulant la geste à une autre dont elle reprendra la versification, ou bien connectant dans une relation de dépendance intertextuelle les exploits de deux personnages liés l'un à l'autre - famille, amitiés, inimitiés -, ou en présentant des caractéristiques physiques ou morales communes. L'auteur peut même déclarer explicitement intégrer sa composition dans la continuité d'une autre : «ce texte est composé à la manière de... » ${ }^{13}$. La nécessaire conscience que le public a de ces filiations textuelles, revendiquées ou non, inscrit le texte dans une tradition et constitue en partie, pour l'auteur, un gage de renommée ou de diffusion de son texte nouveau-né.

Composer sur les affaires de Charlemagne, ou celles de la croisade, c'est de fait s'inscrire dans une tradition. L'écriture cyclique a une cohérence propre. La reconnaissance ou la constitution du cycle est donc le fait de l'auteur, ou de la réception (et éventuellement d'elle seule) - scribe, public savant ou populaire, médiéval ou moderne. Il est caractérisé par les traits communs des textes qui le constituent, ceux-là peuvent être de deux natures : liés au style (lien formel), ou aux arguments (lien thématique).

\section{Les deux cycles français de la croisade}

La littérature médiévale française compte deux cycles de chansons de croisade épiques, l'un ancien, celui qui nous intéresse ici, l'autre plus récent, fruit du renouvellement des poèmes de croisade aux XIV et $\mathrm{XV}^{\mathrm{e}}$ siècles ${ }^{14}$.

Le premier cycle compte treize branches ${ }^{15}$. Son noyau primitif est composé de trois poèmes : la Chanson d'Antioche, les Chétifs et la Conquête

12. Sur la notion de cycle, on pourra voir B. BesamusCA, W. P. Gerritsen, C. Hogetoorn, O. S. H. LIE éd., Cyclification. The Developpement of Narrative Cycles in the Chanson de Geste and Arthurian Romances, Amsterdam, 1994. Voir aussi les réflexions de R. TrACHSLER, Clôtures du cycle arthurien : études et textes, Genève, 1996.

13. Il convient sur ce point de penser, par exemple, au prologue de la Chanson de la Croisade albigeoise.

14. S. DupARC-Quioc, Le Cycle de la Croisade, Paris, 1955, p. 91.

15. R. F. CooK, "Chanson d'Antioche », chanson de geste..., p. 1. 
de Jérusalem, qui nous est connue par l'arrangement qu'en fit, selon la vulgate, Graindor de Douai à la fin du XII siècle. Ce dernier remanie la version primitive de la Conquête de Jérusalem et donne pour la Chanson d'Antioche le nom de l'auteur primitif : Richard le Pèlerin. Graindor, auteur du cycle en quelque sorte, intercale les Chétifs entre la Chanson d'Antioche et la Conquête de Jérusalem, ce qui le conduit à remanier le début de cette dernière pour opérer la soudure. Il faudrait rappeler ici que Robert Francis Cook s'est opposé à cette généalogie, tentant la démonstration, controversée, selon laquelle le texte cyclique d'Antioche est « un original » que rien n'aurait précédé ${ }^{16}$.

Ce noyau dur est parfois précédé dans les témoins par une série de textes qui développent l'histoire du chevalier au cygne comme ancêtre de Godefroi de Bouillon - Chevalier au Cygne, Enfances Cygnes, Enfances Godefroi. Une chanson finale annoncée, sur les débuts de l'Hôpital et le Temple, est perdue. Enfin, ce premier cycle, dans son deuxième état ${ }^{17}$, voit succéder à la Conquête de Jérusalem une série de continuations ${ }^{18}$ allant jusqu'à la mort de Baudoin $1^{\text {er }}$ et le temps de Saladin, la Prise d'Acre et la Mort Godefroi ${ }^{19}$.

Le lien entre la famille de Bouillon et la légende du cygne est assez ancien pour se trouver déjà chez Guillaume de Tyr. C'est là le thème qui sera exploité dans le second cycle des $\mathrm{XIV}^{\mathrm{e}}$ et $\mathrm{XV}^{\mathrm{e}}$ siècles ${ }^{20}$, qui ne comprend que la Chanson du Chevalier au Cygne et de Godefroid de Bouillon, le Baudoin de Sebourc, le Bâtard de Bouillon ainsi que Saladin - dont la version en vers est perdue et qui est seulement connu en prose, comme troisième partie du roman de Jean d'Avesnes ${ }^{21}$.

Rappelons que la Gran Conquista de Ultramar ${ }^{22}$ contient en plus des fragments de la Canso d'Antioca, « la Chanson d'Antioche française, les

16. Ibid., p. 24, 31. R. F. Cook est contre, mais semble isolé et extrémiste dans sa déconstruction.

17. $C f$. les ms. les plus récents, comme Paris, BnF, Arsenal 3139 et fr. 12569.

18. La Chrétienté Corbaran, et seulement dans le ms. Paris, BnF, fr. 12569.

19. S. DuPARC-Quioc, Le Cycle de la Croisade..., p. XIX-XX.

20. R. F. COOK et L. S. CRIST, Le Deuxième Cycle de la croisade. Deux études sur son développement. Les textes en vers. Saladin, Genève, 1972. S. DuPARC-QUIOC, « Les poèmes du deuxième cycle de la croisade : problèmes de composition et de chronologie », Revue d'histoire des textes, 9 (1979), p. 141-181.

21. S. DuparC-Quioc, Le Cycle de la Croisade..., p. 91-97. Cf. aussi R. F. CooK, "Chanson d'Antioche", chanson de geste..., p. 79.

22. Composée à la fin du XIII ${ }^{\mathrm{e}}$ siècle (après 1293) et compilant en quatre livres des faits historiques mêlés de traits épiques, la Gran Conquista de Ultramar reprend et traduit partiellement sa matière de diverses chansons de geste françaises essentiellement, de Guillaume de Tyr et de la Chanson d'Antioche provençale. Voir G. PARIS, « La Chanson d'Antioche provençale et la Gran Conquista de Ultramar ", Romania, 68 (1888), p. 513541 ; ID., «La Chanson d'Antioche provençale et la Gran Conquista de Ultramar (suite)», Romania, 76 (1890), p. 562-591 ; ID., «La Chanson d'Antioche provençale et la Gran 
Chétifs et la Conquête de Jérusalem (telle que nous la possédons jusqu'au chant six), le tout coupé de chapitres de l'Eracles, la traduction française de Guillaume de Tyr, reproduits mot pour $\operatorname{mot}^{23} »$. Suzanne Duparc-Quioc soutenait que, pour les textes français, l'auteur de la Gran Conquista de Ultramar devait disposer de manuscrits très semblables à ceux que nous possédons et avoir procédé de son propre chef aux modifications que l'on y lit. Cela paraît cependant douteux, tant les écarts entre les textes s'accumulent ${ }^{24}$.

Si nous focalisons ici notre réflexion sur les modalités de constitution du cycle de croisade dans la littérature occitane, il faut remarquer que l'exemple du premier cycle de la croisade en français diffère grandement de la constitution du cycle en langue d'oc. En effet, les trois textes français les plus anciens du premier cycle - Antioche, Chétifs, Jérusalem - sont pensés comme éléments d'un cycle par Graindor de Douai qui les articule entre eux en raison de leur cohérence globale pour former le noyau dur de ce premier cycle. Ces trois chansons sont transmises ensemble, dans leur cohérence cyclique, par les manuscrits qui les attestent, et c'est en tant que cycle qu'elles sont immédiatement connues par leur public. C'est là le poète/ compilateur/remanieur médiéval qui, d'emblée, et pour les trois textes, les livre comme appartenant à ce que nous appelons un cycle, effaçant de l'esprit du public l'état antérieur de textes indépendants les uns des autres - et donc non pris dans un processus de cyclisation. C'est là une différence majeure avec la situation du corpus occitan, où l'articulation entre les textes se fait au cas par cas et au fil du temps dans une démarche auctoriale, et où c'est la critique qui, en fin de compte et tenant dans ses mains un ensemble textuel fini, identifie le cycle en tant qu'ensemble - au-delà donc du cas par cas de l'auteur.

\section{Le cycle occitan de la croisade}

À côté du premier cycle français de la croisade, il en existe un autre, du moins c'est notre hypothèse, proprement occitan. Celui-ci, cette fois, ne s'établit pas sur la commune présence d'un personnage, mais sur la reprise à la fois d'une thématique (la croisade, puis la guerre) et d'éléments formels (la versification et la mélodie), déclarés ou implicites (mais dans ce cas évidents). Ce cycle est composé en tant que tel de deux textes, la Canso

23. S. DuPARC-Quioc, Le Cycle de la Croisade..., p. 45.

24. Ibid., p. 46-55. 
d'Antioca et la Chanson de la Croisade albigeoise, auxquels il convient d'adjoindre le Poème de la Guerre de Navarre.

Le plus ancien texte est la Canso d'Antioca ou Chanson d'Antioche occitane, ou provençale, dont Paul Meyer fut le premier éditeur ${ }^{25}$ : un texte de la fin du XII ${ }^{\text {e }}$ siècle, ayant pour sujet la première croisade et le siège d'Antioche de $1098^{26}$. Il est parvenu jusqu'à nous sous forme fragmentaire : seuls 707 vers en sont connus dans un manuscrit conservé à Madrid ${ }^{27}$. Ces vers doivent peut-être être complétés de portions de la castillane Gran Conquista de Ultramar (fin $\mathrm{du} \mathrm{XIII}^{\mathrm{e}}$ siècle) qui transmet en partie des vers de la Canso attestés en occitan et, en d'autres lieux, des passages qui pourraient avoir été empruntés au texte perdu de Gregori Bechada ayant servi de modèle à la version de Madrid qui nous est parvenue.

Le texte occitan conservé est donc une refonte d'une plus ancienne chanson de geste occitane perdue - récit de la première croisade-composée certainement entre 1106 et 1118 par Gregori Bechada sur commande d'Eustorge, évêque de Limoges. Gregori Bechada fut un témoin oculaire des événements. Plaçant l'accent sur les acteurs occitans de cette première croisade, les vers conservés constituent un document d'histoire important pour la connaissance des actions des chevaliers méridionaux et des Italo-Normands, comme Gouffier de Lastours ou Bohémond de Tarente, ce Normand au petit royaume au sud de l'Italie. Bien que Raymond IV de Toulouse soit absent des vers de la Canso d'Antioca conservés, il est probable que ce grand seigneur des pays d'oc appartenant à l'aire culturelle et seigneuriale des attaches du poète y était mentionné.

Cette chanson va servir de modèle - mais dans quelle version ? Celle que l'on connaît, ou celle, primitive et perdue, de Gregori Bechada (sans compter les versions intermédiaires éventuelles) ? - à Guilhem de Tudela pour la composition de sa Chanson de la Croisade albigeoise que continuera un auteur anonyme, laquelle chanson servira elle-même de modèle à Guilhem Anelier de Tolosa, auteur du Poème de la Guerre de Navarre.

La Chanson de la Croisade albigeoise ${ }^{28}$ est ainsi une chanson de geste occitane rapportant les faits de la Croisade albigeoise de 1208 à 1219 et fruit de la composition de deux auteurs successifs. Au premier de ces auteurs,

25. P. MEYER, «Fragment d'une chanson d'Antioche en provençal», Archives de l'Orient latin, 2 (1884), p. 467-509.

26. The Canso d'Antioca: an Occitan epic chronicle of the First Crusade, éd. C. SweEtenham et L. M. PATERson, Burlington, 2003.

27. Ibid. Il s'agit du Codex 117 conservé à la Real Academia de la Historia de Madrid dans les papiers de Jaime de Vilanueva, qui découvrit le texte en un lieu inconnu et alors qu'il était un grand visiteur d'églises espagnoles où il récolta de nombreux manuscrits.

28. La Chanson de la Croisade albigeoise, éd. E. MARTIN-CHABOT, t. I-III, Paris, (19311961) 1960-1973 (2éd.). 
Guilhem de Tudela, a succédé un Anonyme ${ }^{29}$. Le texte, aux prises avec l'actualité immédiate, ou presque, des pays languedociens, est considéré comme une chronique historico-épique. Il est pour ses auteurs canso, gesta et estoria $^{30}$. Composé entre 1209 et 1229 , c'est, dans sa première partie, le récit des exploits des croisés français et de leurs alliés dans la lutte contre les Méridionaux protecteurs d'hérétiques et, dans sa seconde partie, le chant épique de la résistance méridionale «Contra l'orgolh de Fransa ${ }^{31}$ » qui, sous couvert de religion, annexe le pays.

Le clivage entre les deux auteurs se retrouve tant dans l'opinion qui se manifeste dans leurs vers que dans la forme du poème. Le texte de Guilhem, originaire de Navarre, qui comprend les laisses 1 à 131, est composé dans un occitan épique teinté de français, là où son successeur anonyme use d'un languedocien ancré entre Toulouse et Foix.

La Chanson compte 214 laisses, et elle constitue le point d'articulation du triptyque de notre cycle de la Croisade. En effet, Guilhem, qui la commence, déclare composer sur le modèle de la Canso d'Antioca, accrochant directement son texte, par le thème - la croisade -, par la forme et par la revendication explicite, à celui de son prédécesseur. L'Anonyme continuateur faisant de même, ses minimes modifications de la manière de versifier de Guilhem préservent la filiation explicite à la Canso d'Antioca.

De même, la Chanson de la Croisade albigeoise constitue le point d'articulation de ce cycle épique occitan, car c'est à elle, et non plus à l'antique modèle de l'Antioca, que l'auteur du Poème de la Guerre de Navarre (fin du XIII ${ }^{\mathrm{e}}$ siècle), Guilhem Anelier de Toulouse, se réfère et accroche à son tour son œuvre, cette fois implicitement, mais de telle manière qu'aucun doute sur la filiation des deux textes ne peut subsister. Si le Poème n'est pas un récit de croisade, il ne s'attache pas moins à notre cycle en constituant un dernier stade, celui de la croisade devenue guerre - telle que la pense l'Anonyme ${ }^{32}$ !; signe de l'évolution du genre au cours de ce XIII' siècle.

29. Nous tenons pour l'anonymat de l'auteur, malgré l'intérêt de la nouvelle hypothèse de F. ZAMBON, « Una nuova ipotesi sull'autore della seconda parte della Canzone della Crociata albigese », Romance Philology, 70 (2016), p. 267-282. Voir aussi F. ZAMBON, « Guilhem Anelier de Tolosa, Ara farai, no·m puesc tener (BdT 204.1) », Lecturae tropatorum, 8 (2015), p. 1-31.

30. Cf. M. RAguin, Lorsque la poésie fait le souverain. Étude sur la Chanson de la Croisade albigeoise, Paris, 2015, p. 609. Toutes les désignations de l'œuvre se trouvent dans la partie attribuée à Guilhem de Tudela. Néanmoins, si l'Anonyme ne nomme pas son texte, sa manière de se couler (fond et forme) dans la narration du premier nous invite à penser qu'il assume, malgré ce qu'il remettra en question, cette première écriture.

31. La Chanson..., t. III, laisse 213, v. 120 et laisse 214, v. 1 a.

32. Cf. M. RaGUIN, Lorsque la poésie fait le souverain..., p. 610-612. 
Le Poème de la Guerre de Navarre est connu, dans ses deux principales éditions, par les travaux de Francisque Michel $^{33}$, son premier éditeur scientifique - laissons de côté la publication navarraise par Don Pablo Ilarregui ${ }^{34}$, et plus récemment par la transcription et le fac-similé du manuscrit avec notes d'édition et étude, ainsi que traduction en castillan et en basque par Maurice Berthe, Ricardo Cierbide, Xabier Kintana, et Julián Santalo ${ }^{35}$.

Ce texte redécouvert au XIX ${ }^{\mathrm{e}}$ siècle est aujourd'hui conservé ${ }^{36}$ dans un manuscrit unique à la Bibliothèque de la Real Academia de la Historia de Madrid, mais il semble qu'ait existé au moins une autre copie aujourd'hui perdue $^{37}$. Ce poème fait le récit de la guerre civile qui toucha Pampelune et le Royaume de Navarre en 1276 et 1277 dans un contexte de succession difficile, à la mort en 1274 du roi Henri I. Ce dernier appartenait à la dynastie de Champagne, qui régnait depuis la mort, en 1234, de Sanche VII, alors que son héritière Jeanne n'était âgée que d'un $a^{38}{ }^{38}$ Les revendications sur la Navarre ne tardèrent pas à venir d'Aragon et de Castille, qui trouvèrent un fort appui chez les populations de la ville dans laquelle s'opposaient, d'une part, les Francos, essentiellement occitans, réunis dans les faubourgs de Saint-Cernin et de Saint-Nicolas, libres de certaines impositions mais navarrais depuis déjà plusieurs générations et, d'autre part, les Navarrais historiques, jaloux des « deux poids, deux mesures » maintenus par la royauté franque. Cette guerre civile, selon l'expression de Francisque Michel, fut avant tout un conflit social ${ }^{39}$. La résistance navarraise fut matée en 1277. Le roi de France Philippe le Hardi, appelé au secours, nomma un nouveau gouverneur venu de Toulouse (où il était depuis 1272), le sénéchal Eustache de Beaumarchais, pour le rétablissement de la paix. Eustache de Beaumarchais emmenait avec lui un individu appelé Guilhem Anelier de Toulouse (qui vit le départ de Saint Louis à Aigues Mortes en 1270 selon ses dires), homonyme, si ce n'est parent, du troubadour du même nom (mais

33. GuILHEM ANELIER de Toulouse, Histoire de la guerre de Navarre en 1276 et 1277 , éd. F. MicheL, Paris, 1856.

34. La Guerra civil de Pamplona, Poema escrito en versos provenzales por Guillermo Aneliers de Tolosa de Francia, éd. P. ILAREGUI, Pampelune, 1847.

35. Guilhem Anelier de Tolosa, La Guerra de Navarra : Nafarroako Gudua, éd. et trad. M. Berthe, R. Cierbide, X. KintanA, J. SANTALO., 2 vol. , Gobierno de Navarra, 1995.

36. Ibid., p. 31. Cote 9.4923 .

37. Ibid., p. 38.

38. Ibid.,p. 58-59.

39. C'est la théorie de Charles Higounet, reprise par M. Berthe, R. Cierbide, X. Kintana et J. Santalo dans leur étude préliminaire à l'édition de La Guerra de Navarra..., p. 57 et sq. Cf. C. Higounet, «Une relecture de Guillaume Anelier », Annales du Midi : revue archéologique, historique et philologique de la France méridionale, 97 (1985), Structures agraires et vie rurale, p. 75-80. 
qui ne doit pas être confondu avec lui) ${ }^{40}$. Guilhem Anelier devint l'auteur du poème relatant cette " guerre de Navarre ». Venu de la ville tant chantée dans la Chanson de la Croisade albigeoise, il en connaissait le poème et en avait d'ailleurs peut-être, dans ses papiers, une copie ${ }^{41}$; et c'est sur le modèle de sa deuxième partie qu'il composa, percevant les traits communs aux deux guerres. Le passage du temps, la mutation des conflits au fil du $\mathrm{XIII}^{\mathrm{e}}$ siècle, qui dans sa seconde partie allait réserver la croisade à l'outremer, le filtre de l'Anonyme sur la croisade devenue guerre et, à coup sûr, le prestige comme la bonne connaissance du modèle littéraire de la Chanson allaient l'emporter sur les différences de point de vue (irréconciliables) qu'expriment les deux textes sur les Français.

Il est à noter que, dans la continuité de la résolution du conflit albigeois, le Poème témoigne d'une autre forme d'héritage : les comtes de Foix, d'Armagnac, de Bigorre et les vicomtes de Béarn marchent avec le connétable de France, Humbert de Beaujeu, et Robert d'Artois ; Occitans et Français, ennemis irréductibles selon la polarisation de la désormais vieille Chanson de la Croisade albigeoise, marchent côte à côte ${ }^{42}$. Là encore, à travers son récit des événements, le Poème témoigne de l'histoire et de ses revirements.

\section{À partir du cas occitan, quelques remarques}

Le cas de la constitution d'un cycle de la croisade en occitan avec deux chansons de croisade proprement dites, celle d'Antioche relative à la première croisade, et celle contre les Albigeois, auxquelles il faut adjoindre la chanson sur la Guerre de Navarre, mérite une étude détaillée; nous faisons là quelques remarques. Il y a en effet matière à examiner une série

40. $C f$. en dernière instance F. ZAMBON, « Guilhem Anelier de Tolosa... ». On verra néanmoins avec intérêt les travaux précédents de R. Straub.

41. Cette hypothèse n'est pas à rejeter d'emblée. En effet, le texte de la Chanson de la Croisade a circulé, c'est certain. À côté du seul manuscrit complet dont nous ayons trace, plusieurs extraits de manuscrits, perdus ou récemment retrouvés, ainsi que des attestations de vers du poème dans d'autres œuvres postérieures, montrent que le texte a circulé dans les environs de Toulouse, avec parfois des variantes textuelles significatives. On pensera à la découverte récente de deux fragments de bifeuillets de la Chanson dans un incunable de la bibliothèque municipale de Toulouse par T. Falmagne, vestige de la Bibliothèque des Dominicains de Toulouse. $C f$. T. FALMAGNE, «Fragments et bibliothèques médiévales à Toulouse: vestiges mineurs, signes de bibliothèques majeures disparues », Cahier de Fanjeaux, 51 (2016), Culture religieuse méridionale. Les manuscrits et leur contexte artistique, p. 349-413.

42. Il est évident que le texte de l'Anonyme ne parlait pas d'Occitans en tant que tels, tout comme sa désignation de ce que sont les Français serait à étudier dans le détail. 
de procédés permettant, du point de vue des auteurs, de construire le lien d'un texte à l'autre; et donc de constituer le cycle.

La Canso d'Antioca est, pour le cycle de la croisade en occitan, le texte premier. C'est le cas du point de vue de l'histoire puisque celui-ci est le plus ancien - il date de la fin du XII ${ }^{\mathrm{e}}$ siècle - et parce que, dans la chronologie des ancrages intertextuels qui établissent le cycle, cette chanson est le modèle du deuxième maillon, lequel servira à son tour de modèle au troisième.

Cette Chanson d'Antioche provençale, dans les 707 vers conservés, est composée de laisses d'alexandrins monorimes. Chaque laisse se termine par un hexasyllabe, petit vers à rime féminine isolée, selon un procédé que l'on trouve aussi dans la tradition française ${ }^{43}$. Ici, ce vers ne rime ni avec sa laisse, ni avec la suivante; de même, il n'établit pas de système capfinit.

La Chanson de la Croisade albigeoise est la deuxième dans la chronologie du cycle. C'est avec sa rédaction sur le modèle de la première que commence l'existence d'un cycle de croisade occitan. Son premier auteur, Guilhem de Tudela, exprime délibérément, à la fin de son prologue ${ }^{44}$, c'est-à-dire au tout début de la deuxième laisse (vers 1 à 3), que : "Senhors esta canso es faita d'aital guia / Com sela d'Antiocha e ayssi·s versifia / E s'a tot aital so qui diire lo sabia ${ }^{45} »$ ( «Seigneurs, cette chanson est faite comme celle d'Antioche et se versifie de la même manière, et a la même mélodie pour qui savait la dire »). Guilhem situe ainsi sa chanson dans un héritage, celui d'une chanson d'Antioche dont il dit explicitement qu'elle est son modèle pour ce qui touche à la versification et la mélodie. Remarquons, au sujet du $s o^{46}$, que Guilhem signe ainsi son instruction et sa qualité de poète du renouveau à partir de classiques; le so de l'Antiocha n'est plus forcément connu, et ne le fut pas de tous « qui diire lo sabia ». On peut même se demander si l' «aital guia » ne recouvre pas aussi la nature du conflit, c'est-à-dire la croisade. Rappelons en effet que, chez Guilhem, et contrairement à son Continuateur anonyme, la guerre en Languedoc est crozada, là où l'autre verra surtout la guerra. Cette influence des idéologies, qui est exprimée à travers la désignation du conflit, a certainement une influence sur la constitution du cycle et le contenu des œuvres qui s’y rattachent : Guilhem - dont on dit pourtant qu'il est presque chroniqueur -

43. A. RonCAGLIA, «Petit vers et refrain dans les chansons de geste », La Technique littéraire des chansons de geste. Actes du Colloque de Liège (septembre 1957), Paris, 1959, p. 141-159.

44. Outre l'édition de E. Martin-Chabot, on verra, pour ce prologue, l'édition de M. RAGUiN-BARTHELMEBS, «Problèmes de transmission textuelle et d'interprétation dans l'épique: le cas du prologue de la Chanson de la Croisade albigeoise. Édition critique synoptique », Medioevo romanzo, 40 (2016), p. 371-396.

45. M. RAgUin-BARTHELMEBS, « Problèmes de transmission textuelle... », p. 392.

46. Le so (son), terme de la lyrique occitane, serait pour nous la mélodie, l'air. 
compose une chanson de croisade épique sur le modèle de celle d'Antioche, chanson de croisade absolue par la nature du conflit et le lieu où il se déroule, alors que l'Anonyme voit dans l'affaire albigeoise, et pour des raisons bien compréhensibles, surtout une guerre (désamorçant ainsi l'aspect de guerre sainte) ; et c'est à travers cette lecture du conflit, la guerre, que Guilhem Anelier peut trouver légitime de couler son œuvre à la suite du cycle ${ }^{47}$.

Guilhem de Tudela s'appuie donc sur le modèle de la Canso d'Antioca pour composer sa propre canso, son propre récit de croisade. Il y a lieu de se demander si cette chanson «d'Antiocha » à laquelle il se réfère, certainement occitane - c'est le contexte comme le fait que l'on connaisse une attestation de cette chanson en langue d'oc qui nous porte à le croire -, correspond à la même version du texte que celle que nous connaissons, ou au texte de Gregori Bechada, ou encore à une autre refonte de celui-ci. On attirera l'attention sur le fait qu'il n'aurait pas été évident qu'il s'agisse là d'un texte en occitan, pour un critique contemporain, si une canso d'Antioca occitane n'avait pas été retrouvée. Cela d'autant plus qu'existe un texte français bien connu et que, confronté à une telle assertion de la part d'un auteur, et hors de présence d'un témoin occitan, l'esprit du critique part directement chercher la source du côté français. C'est là, à nos yeux, un certain avertissement méthodologique : en présence d'une référence littéraire dans un texte occitan, y compris lorsqu'il s'agit de parler d'un long texte narratif, on peut légitimement s'interroger sur le fait que l'auteur qui mentionne ce texte a connu la version française que nous connaissons, et vers laquelle notre esprit se tourne presque automatiquement, ou si une version en langue d'oc a existé, et s'il n'y a donc pas là un intertexte à prendre en compte.

C'est en tout cas un texte de cette même tradition de chansons d'Antioche provençales que Guilhem prend pour modèle. En effet, sa chanson est elle aussi composée de laisses d'alexandrins monorimes, chaque laisse se terminant par un hexasyllabe. C'est ici que se situe l'originalité de Guilhem vis-à-vis de la Canso d'Antioca que nous connaissons, qu'elle ait été son modèle ou pas : l'hexasyllabe chez lui, selon la capcaudada troubadouresque, ne rime pas avec la laisse à laquelle il appartient mais donnera la rime de la suivante. Pour ces raisons, Paul Meyer, premier éditeur de la Chanson d'Antioche en langue d'oc et éditeur aussi de la Chanson de la Croisade albigeoise, se demandait déjà si la Canso d'Antioca à laquelle se réfère Guilhem de Tudela est bien celle que nous connaissons ${ }^{48}$.

Dans la première partie de la Chanson de la Croisade albigeoise, le fait de se placer dans la continuité de la Canso d'Antioca est donc revendiqué, ce qui rappelle au passage qu'une geste, aux yeux de son

47. Pour rappel, $c f$. M. RAGUIN, Lorsque la poésie fait le souverain..., p. 610-612.

48. P. MEYER, « Fragment d'une chanson d'Antioche en provençal... », p. 468-469. 
auteur, se versifia et est dotée d'un so. Dans la partie de la Chanson de la Croisade albigeoise écrite par l'Anonyme, à partir de la laisse 132, il faut rappeler que, parmi les modifications que ce second auteur applique à son texte - de ton, d'opinion, etc. -, il y en a une, formelle, de premier plan : celle qui touche à l'hexasyllabe en finale de laisse. La partie anonyme du texte adopte en effet le système de la capfinida troubadouresque auquel elle combine, comme par retour à un modèle qui s'approcherait de ce que l'on connaît de la Canso d'Antioca, un petit vers final - mais sans rime féminine - qui ne rime ni avec sa laisse ni avec la suivante, mais qui donnera, remanié, le premier hémistiche ou le premier vers suivant de la laisse successive. Cet enchaînement capfinit pourrait être une manière de combiner tradition troubadouresque et référence à la Canso d'Antioca telle que l'auteur anonyme la connaîtrait. Si cela est bien incertain, cette hypothèse tendrait à montrer que l'Anonyme était soucieux de souligner sa culture littéraire et son inscription dans une filiation poétique.

C'est cette technique d'enchaînement capfinit que l'auteur du Poème de la Guerre de Navarre reprendra. Guilhem Anelier de Tolosa va ainsi inscrire à son tour son texte, par la forme poétique qu'il lui donne, dans une dépendance claire envers la Chanson de la Croisade albigeoise, en fait aux deux parties du texte; celle de Guilhem de Tudela et celle de l'Anonyme. Contrairement à Guilhem, l'auteur du Poème de la Guerre de Navarre ne déclare pas suivre de modèle, ou composer « à la manière de ». Mais il le fait, et le fait dans les grandes lignes comme dans les détails. On remarquera, dans cette perspective, un prologue très similaire ; le rappel de la bataille de Las Navas de Tolosa (Chanson de la Croisade albigeoise, laisse 6 ; Poème de la Guerre de Navarre, laisses 2-3), de très nombreuses reprises d'hémistiches ou de formules présentes dans la seconde partie de la Chanson de la Croisade albigeoise ${ }^{49}$; enfin et surtout - comme si le petit vers final était le fil conducteur pour la constitution de ce cycle épique - l'adoption, à l'identique cette fois, de la technique d'enchaînement par hexasyllabe isolé dans un système de capfinidas, conformément à la partie anonyme de son modèle. Le Toulousain Guilhem Anelier connaissait bien la Chanson continuée par l'Anonyme, et pas seulement le premier texte de Guilhem de Tudela.

Cette remarque est de grande importance pour la question du cycle de la croisade en occitan. On pourrait dire en effet que le Poème de la Guerre de Navarre n'est pas une chanson de croisade ; c'est vrai. Mais c'est bien un poème de guerre méridionale (l'auteur vient de Toulouse), composé sur le modèle de la Chanson de la Croisade albigeoise, elle-même composée sur le modèle de la Canso d'Antioca. Et c'est parce que l'auteur du Poème de la

49. Pour une première approche, $c f$. M. RAGUIN, Lorsque la poésie fait le souverain..., p. 104-109. L'inventaire complet de ces intertextualités et emprunts reste à faire. 
Guerre de Navarre est un lecteur de la Chanson de la Croisade albigeoise, chanson de croisade épique donc, à travers le texte de l'Anonyme - qui fait, dans sa narration, de la croisade de Guilhem de Tudela une guerre politique et non plus tellement, plus du tout en fait, à justification religieuse - que Guilhem Anelier peut choisir à son tour le modèle de la Chanson de la Croisade albigeoise au-delà de la question de la diffusion et de la qualité littéraire du modèle. Il y a, pour lui, une connivence des thèmes qui permet une telle filiation littéraire, et à l'évidence, celle-ci est seulement rendue possible par sa lecture de l'œuvre terminée par l'Anonyme - et ce quand bien même il aurait appartenu à sa descendance selon l'hypothèse de Zambon : il y a là, pour nous, avant tout, un choix esthétique de littérateur. Cela d'autant plus que le rapprochement idéologique n'est guère possible : le texte de Guilhem Anelier n'a rien d'un plaidoyer anti-français.

Guilhem Anelier ne justifie pas son choix - d'ailleurs, à la fin du $\mathrm{XIII}^{\mathrm{e}}$ siècle, sa connaissance de la Chanson de la Croisade albigeoise à Toulouse et en Languedoc était à coup sûr livresque, étant donné le climat après la défaite de 1229. Il faut que ce soient l'éclat du poème et l'art qu'il reconnaît à son auteur (dans ce qui est pour nous la seconde partie puisqu'il adopte ses formules et son style) qui justifient le choix de ce modèle et le fait qu'il prenne part, à son tour, à ce qui aurait été certainement à ses yeux un cycle épique occitan de la guerre plutôt que de la croisade.

Il est remarquable qu'il n'y ait pas dans le domaine occitan de récit de croisade isolé du cycle, de même qu'il n'y a pas de manuscrit cyclique pour la croisade, contrairement aux deux Roland occitans, Ronsasvals et Roland à Saragosse. Les récits de croisade que sont la Canso d'Antioca et la Chanson de la Croisade albigeoise, et après la croisade le récit de guerre du Poème de la Guerre de Navarre (selon la logique de son auteur vis-àvis de la partie anonyme de Chanson de la Croisade albigeoise), se font sur le modèle formel du précédent, constituant ainsi le cycle. Une écriture cyclique qui, en définitive, s'établit d'abord par la reproduction déclarée d'une forme littéraire (versification et mélodie), et d'un thème (la croisade) de la Canso d'Antioca à la Chanson de la Croisade albigeoise, puis de la Chanson de la Croisade albigeoise au Poème de la Guerre de Navarre, avec là encore la reproduction implicite d'une forme guidée par la thématique guerrière, et même de franches reprises à l'identique d'hémistiches entiers, devenus formulaires pour qui connaîtrait les deux textes.

La non-déclaration de son modèle par Guilhem Anelier de Toulouse, alors même qu'il semble composer avec celui-ci sous les yeux tant certains vers sont communs aux deux textes, porte à penser que son silence est lié à la mauvaise presse qu'avaient les opinions véhiculées par la Chanson de la Croisade albigeoise après la défaite de 1229 et les révoltes successives ratées. En somme, il reprend le modèle littéraire, ses bonnes formules, son art, mais en le vidant de sa substance subversive. 
Pour conclure en quelques mots ces réflexions, il semble que l'écriture dans le cadre des chansons de croisade, ou des cycles de chansons de croisade français ou occitan, est, du point de vue de leurs auteurs et/ou remanieurs, celle d'une cohérence d'ensemble. Le cycle, ou son noyau dur, peut être établi matériellement par le biais de l'objet qu'est le livre manuscrit ; les chansons peuvent être remaniées pour faciliter l'enchaînement et la cohérence - avec un souci d'historicité à ne pas négliger. C'est le modèle français pour les chansons antiques du premier cycle de la croisade. Dans ce cas, comme dans leurs continuations constituant le reste du cycle, c'est la matière et les personnages principaux qui font la cohérence, au-delà de traits stylistiques communs aux grands répertoires des chansons de geste.

Le cas est tout autre dans le domaine occitan, et à nos yeux plus instructif sur le processus de constitution du cycle par l'auteur lui-même : il inscrit sa chanson dans une filiation littéraire par la forme, le style, plutôt que par les personnages ; la matière croisée ou a minima guerrière étant un prérequis pour la cohérence thématique autorisant l'auteur à y inscrire son œuvre du point de vue de la tradition. La chanson de croisade s'écrit en cycle en occitan aussi, c'est très clair : soit que l'auteur revendique clairement son modèle : la Chanson de la Croisade albigeoise vis-à-vis de la Canso d'Antioca; soit que celui-ci apparaisse très nettement aux connaisseurs des deux textes : le Poème de la Guerre de Navarre par rapport à la Chanson de la Croisade albigeoise.

Ainsi, écrire ou composer à la manière d'un autre est l'expression claire d'une volonté de l'auteur, en occitan du moins - mais on pourrait dire, pour le noyau dur français, que c'est aussi le cas pour le remanieur qui, à sa manière, fait œuvre auctoriale en réorientant la lecture d'œuvres rassemblées ${ }^{50}$. L'intérêt de la cyclisation, au-delà des avantages évidents du remploi d'une mélodie ou d'un style de versification connus qui aideront le nouveau texte - et son auteur, même anonyme - à trouver le succès, réside en premier lieu, selon nous, dans cette dialectique du trobar qu'il convient de ne jamais perdre de vue, cette compétition littéraire entre poètes qui se mesurent les uns aux autres : composer à la manière d'un autre, c'est tenter de le dépasser sur son propre terrain.

Marjolaine Raguin-Barthelmebs - Marie-Curie CoFund Postdoctoral Fellow, Université de Liège (Belgique)

50. A fortiori, mais pas seulement, parce que la compilation implique souvent un travail de modifications textuelles en vue de l'arrangement des œuvres entre elles. 


\section{Quand la chanson de croisade se compose en cycle. Expériences d'écritures françaises et occitanes}

La présente contribution propose une réflexion sur les processus de cyclisation de l'écriture des chansons de croisade épiques. S'intéressant d'abord au corpus français bien connu pour étudier ensuite celui plus confidentiel de langue occitane, l'auteure propose d'identifier un cycle épique de la croisade en langue d'oc composé des trois textes de la Canso d'Antioca, de la Chanson de la Croisade albigeoise, et du Poème de la guerre de Navarre. Elle en étudie les modalités propres de constitution, soulignant les spécificités de ces écritures occitanes au regard des connaissances de la cyclisation d'un cycle analogue en français; insistant pour le cas occitan sur le rôle central du texte de la Chanson de la Croisade albigeoise dans ses deux parties.

Chanson d'Antioche occitane, Chanson de la Croisade albigeoise, chansons de croisade épiques, écriture cyclique, Guilhem Anelier, Guilhem de Tudela/ Guillaume de Tudèle, Poème de la guerre de Navarre.

\section{When the Crusade Song is Composed in Cyclic Form. French and Occitan Experiences of Writing}

This paper considers the process of cyclic writing in epic crusade songs. Examining first the well-known French corpus, and then the more unfamiliar Occitan texts, the author proposes to identify an Occitan Crusade cycle composed of three texts: the Occitan Canso d'Antioca, the Albigensian Crusade Song, and the Navarra War Poem. She studies how this cycle is formed and by which critical criteria it can be identified. She stresses the specificities of this Occitan corpus and its cyclization in comparison to the French one, highlighting the pivotal role that the two parts of the Albigensian Crusade Song had in establishing the Occitan Crusade cycle.

Albigensian Crusade Song, Cyclic form, Epic crusade songs, Guilhem Anelier, Guilhem de Tudela/Guillaume de Tudèle, Navarra War Poem, Occitan Canso d'Antioca. 